University of Texas at El Paso

ScholarWorks@UTEP

Relationship Between Measurement Results and Expert Estimates of Cumulative Quantities, on the Example of Pavement Roughness

\author{
Edgar Daniel Rodriguez Velasquez \\ The University of Texas at El Paso, edrodriguezvelasquez@miners.utep.edu \\ Carlos M. Chang Albitres \\ The University of Texas at El Paso, cchangalbitres2@utep.edu \\ Vladik Kreinovich \\ The University of Texas at El Paso, vladik@utep.edu
}

Follow this and additional works at: https://scholarworks.utep.edu/cs_techrep

Part of the Computer Sciences Commons

Comments:

Technical Report: UTEP-CS-19-43

\footnotetext{
Recommended Citation

Rodriguez Velasquez, Edgar Daniel; Chang Albitres, Carlos M.; and Kreinovich, Vladik, "Relationship Between Measurement Results and Expert Estimates of Cumulative Quantities, on the Example of Pavement Roughness" (2019). Departmental Technical Reports (CS). 1327.

https://scholarworks.utep.edu/cs_techrep/1327
}

This Article is brought to you for free and open access by the Computer Science at ScholarWorks@UTEP. It has been accepted for inclusion in Departmental Technical Reports (CS) by an authorized administrator of ScholarWorks@UTEP. For more information, please contact Iweber@utep.edu. 


\title{
Relationship Between Measurement Results and Expert Estimates of Cumulative Quantities, on the Example of Pavement Roughness
}

\author{
Edgar Daniel Rodriguez Velasquez ${ }^{1,2}$, Carlos M. Chang Albitres ${ }^{2}$, \\ and Vladik Kreinovich ${ }^{3}$ \\ ${ }^{1}$ Department of Civil Engineering \\ Universidad de Piura in Peru (UDEP) \\ Av. Ramón Mugica 131, Piura, Peru, edgar.rodriguez@udep.pe \\ ${ }^{2}$ Department of Civil Engineering \\ ${ }^{3}$ Department of Computer Science \\ University of Texas at El Paso, $500 \mathrm{~W}$. University \\ El Paso, TX 79968, USA \\ edrodriguezvelasquez@miners.utep.edu, cchangalbitres2@utep.edu, \\ vladik@utep.edu
}

\begin{abstract}
In many practical situation, we are interesting in values of cumulative quantities - e.g., quantities that describe the overall quality of a long road segment. Some of these quantities we can measure, but measuring such quantities requiring measuring many local values and is, thus, expensive and time-consuming. As a result, in many cases, instead of the measurement, we reply on expert estimating such cumulative quantities on a scale, e.g., from 0 to 5 . Researchers have come up with an empirical formula that provides a relation between the measurement result and a 0 -to- 5 expert estimate. In this paper, we provide a theoretical explanation for this empirically efficient formula.
\end{abstract}

\section{Formulation of the Problem}

Cumulative quantities. Many physical quantities can be measured directly: e.g., we can directly measure mass, acceleration, force, etc. However, in many practical applications, we are interested in cumulative quantities that combine values corresponding to different moments of time and/or different locations. For example, when we are studying public health or pollution or economic characteristics, we are often interested in characteristics describing the whole city, the whole region, the whole country, etc. 
Formulation of the problem. Cumulative characteristics are not easy to measure. To measure each such characteristic, we need to perform a large number of measurements, and then use an appropriate algorithm to combine these results into a single value. Since such measurements are complicated, in many applications, we have to supplement the difficult-to-get results of measuring some cumulative quantities with expert estimates of other such quantities.

As a result, we often have both measurement results corresponding to some situations and expert estimates corresponding to other situations. To process such data, it is desirable to describe both estimates in the same scale. For this, it is desirable to come up with a way to estimate the actual value of the corresponding quantity based on the corresponding expert estimate, and vice versa.

Case study: estimating pavement roughness. As a case study, we consider the problem of estimating the pavement roughness. This is an example of cumulative quantity: we are interested in gauging the quality of the whole road segment, a road segment that may includes local parts with different roughness characteristics.

Estimating road roughness is an important problem. Indeed, road pavements need to be maintained and repaired. Both maintenance and repair are expensive. So, it is desirable to estimate the pavement roughness as accurately as possible:

- if we overestimate the road roughness, we will waste money and other resources on road segments which are in reasonably good shape, at the expense of other segments which may need maintenance or repair;

- if we underestimate the road roughness, the road segment will be left unrepaired and deteriorate even more - as a result of which the cost of its future repair will skyrocket.

The standard way to measure the pavement roughness is to use the International Roughness Index (IRI); see, e.g., $[4,5,6,8]$. This measure of roughness is recommended by the US standards $[4,5,6]$.

Crudely speaking, IRI describes the effect of the pavement roughness on a standardized model of a vehicle. Measuring IRI is not easy, because the real vehicles differ from this standardized model. As a result, we measure roughness by some instruments and use these measurements to estimate IRI. For example, we can:

- perform measurements by driving an available vehicle along this road segment,

- extract the local roughness characteristics from the effect of the pavement on this vehicle, and then

- use these extracted characteristics to estimate the effect of the same pavement on the standardized vehicle. 
In view of this difficulty, in many cases, practitioners rely on expert estimates of the pavement roughness. The corresponding measure - estimated on a scale from 0 to 5 - is known as the Present Serviceability Rating (PSR); see, e.g., [3, 7].

Empirical relation between measurement results and expert estimates, on the example of pavement roughness. The empirical relation between PSR and IRI is described by the following formula:

$$
\mathrm{PSR}=5 \cdot \exp (-0.0041 \cdot \mathrm{IRI})
$$

This formula was first proposed by B. Al-Omari and M. Darter in [2].

From the qualitative viewpoint, this formula makes perfect sense:

- when the pavement is perfect, then IRI is 0 , so, according to this formula, the subjective estimate PSR get the higher possible value of 5 ;

- on the other extreme, if the pavement is in very bad condition, then IRI is very large, so PSR is close to 0 - as it should be.

In general, the dependence of PSR on IRI should be motonotic (namely, it should be decreasing). However, from the quantitative viewpoint, it is not clear why namely this formula works so well - and it indeed works much better than many previously proposed alternative formulas.

What we do in this paper. In this paper, we propose a possible explanation for the above empirical formula. This explanation will be general: it will apply to all possible cases of cumulative quantities. We will come up with a general formula $y=f(x)$ that describes how a subjective estimate $y$ of a cumulative quantity depends on the result $x$ of its measurement. As a case study, we will use gauging road roughness.

\section{Our Explanation}

Main idea: both measurement results and subjective estimates can have different values. In general, the numerical value of a subjective estimate depends on the scale. In road roughness estimates, we usually use a 0-to- 5 scale. In other applications, it may be more customary to use 0 -to- 10 or 0 -to- 1 scales. A usual way to transform between the two scales is to multiply all the values by a corresponding factor. For example, to transform an estimate from a 0 -to-10 scale to a 0 -to- 1 scale, we can simply multiply all the values by $\lambda=0.1$. In other transitions, we can use transformations $y \rightarrow \lambda \cdot y$ with different re-scaling factors $\lambda$.

There is no major advantage in selecting a specific space. So, we can conclude that subjective estimates are defined modulo such a re-scaling transformation

$$
y \rightarrow \lambda \cdot y \text {. }
$$


At first glance, the result of measuring a cumulative quantity may look uniquely determined (in contrast to subjective estimates). However, a detailed analysis shows that there is some non-uniqueness here as well. Indeed, the result of a cumulative measurement comes from combining values measured at different moments of time and/or values corresponding to different spatial locations. Even when for each individual measurement, the probability of a sensor's malfunction may be low, still, when we perform a large number of measurements, some of them bound to be caused by such malfunctions and are, thus, outliers. It is well known that even a single outlier can drastically change the average. So, to avoid such influence, the usual algorithms for computing the values of the cumulative properties do not simply combine all the measurement results, they first filter out possible outliers.

This filtering out is never an exact science: we can set up slightly different thresholds for detecting an outlier, slightly different threshold for allowed number of remaining outliers, etc. So, in addition to the computation result that only takes actual signals into account, with a different setting, we may get a somewhat different result, in which a few outliers are also taken into account. If the average value of an outlier is $L$ and the average number of such outliers is $n$, then the second scheme, in effect, adds a constant $n \cdot L$ to the cumulative value computed by the first scheme. Yes, there is also some random deviation, but when the number $n$ is reasonably large, then, due to the Large Numbers theorem, these deviations average out and we get approximately the mean value (see, e.g., [9]) - just like when we flip a coin many $(N)$ times, the overall number of times when it falls head will be close to $0.5 \cdot N$.

From this viewpoint, the measured value of a cumulative quantity is defined modulo an addition of some value, i.e., modulo a transformation $x \rightarrow x+a$ for some constant $a$.

Motivation for invariance. As we have just mentioned, the measurement results are determined uniquely modulo a shift $x \rightarrow x+a$. We do not know exactly what is the ideal threshold, so we have no reason to select a specific shift as ideal. It is therefore reasonable to require that the desired formula $y=f(x)$ not depend on the choice of such a shift, i.e., that the corresponding dependence not change if we simply replace $x$ with $x^{\prime}=x+a$.

Of course, we cannot just require that $f(x)=f(x+a)$ for all $x$ and all $a$, because in this case, the function $f(x)$ will simply be a constant (and we will have a meaningless conclusion that the subjective value $y$ does not depend on the measurement result at all). But this is clearly not how invariance is usually defined. For example, for many physical interactions, there is no fixed unit of time, so formulas should not change if we simply change a unit for measuring time, i.e., replace the original numerical value $t$ with a new value $t^{\prime}=\lambda \cdot t$, where $\lambda$ is the ratio between the old and the new units. However, this does not mean that, e.g., the formula $d=v \cdot t$ that relates the distance $d$, the velocity $v$, and the time $t$ should not change if we simply replace the time $t$ with the new time $\lambda \cdot t$. To make this formula true when time is measured in the new units, we may need to also appropriately change the units of other related quantities. 
In the above example, we need to appropriately change the unit for measuring velocity, so that not only time units are changed, e.g., from hours to second, but velocities are also changed from $\mathrm{km} /$ hour to $\mathrm{km} / \mathrm{sec}$.

In line with this reasoning, if we re-scale $x$, the formula $y=f(x)$ should remain valid if we appropriately re-scale $y$. As we have mentioned earlier, possible re-scalings of the subjective estimate $y$ have the form $y \rightarrow y^{\prime}=\lambda \cdot y$. Thus, invariance in this case means that for each $a$, there exists an appropriate value $\lambda(a)$ depending on $a$ for which $y=f(x)$ implies that $y^{\prime}=f\left(x^{\prime}\right)$, where $x^{\prime}=x+a$ and $y^{\prime}=\lambda \cdot y$.

So, we arrive at the following definition.

\section{Definitions and the Main Result}

Definition 1. We say that a monotonic function $f(x)$ is unit-invariant if for every real number $a$, there exists a positive real number $\lambda(a)$ for which, for each $x$ and $y$, if $y=f(x)$, then $y^{\prime}=f\left(x^{\prime}\right)$, where $x^{\prime} \stackrel{\text { def }}{=} x+a$ and $y^{\prime} \stackrel{\text { def }}{=} \lambda(a) \cdot y$.

Proposition 1. A function $f(x)$ is unit-invariant if and only if it has the form $f(x)=C \cdot \exp (-b \cdot x)$ for some $C$ and $b$.

Conclusion. For the case study of gauging road roughness, this result explains the empirical success of the formula proposed in [2].

Proof. It is easy to check that every function $y=f(x)=C \cdot \exp (-b \cdot x)$ is indeed unit-invariant. Indeed, for each $a$, we have

$f\left(x^{\prime}\right)=f(x+a)=C \cdot \exp (-b \cdot(x+a))=C \cdot \exp (-b \cdot x-b \cdot a)=\lambda(a) \cdot C \cdot \exp (-b \cdot x)$,

where we denoted $\lambda(a) \stackrel{\text { def }}{=} \exp (-b \cdot a)$. Thus here, indeed, $y=f(x)$ implies that

$$
y^{\prime}=f\left(x^{\prime}\right)
$$

Vice versa, let us assume that the function $f(x)$ is unit-invariant. Then, for each $a$, the condition $y=f(x)$ implies that $y^{\prime}=f\left(x^{\prime}\right)$, i.e., that $\lambda(a) \cdot y=$ $f(x+a)$. Substituting $y=f(x)$ into this equality, we conclude that

$$
f(x+a)=\lambda(a) \cdot f(x) .
$$

It is known (see, e.g., [1]) that every monotonic solution of this functional equation has the form $f(x)=C \cdot \exp (-b \cdot x)$, for some $C$ and $b$.

The proposition is proven.

\section{Acknowledgments}

This work was partially supported by the Universidad de Piura in Peru (UDEP) and by the US National Science Foundation via grant HRD-1242122 (CyberShARE Center of Excellence). 


\section{References}

[1] J. Aczél and J. Dhombres, Functional Equations in Several Variables, Camridge University Press, Cambridge, UK, 2008.

[2] B. Al-Omari and M. Darter, "Relationship between International Roughness Index and Present Serviceability Rating", Transportation Research Record, 1994, No. 1435, pp. 130-136.

[3] American Association of State Highway and Transportation Officials (AASHTO), AASHTO Guide for Designing Pavement Structures, AASHTO, Washington, DC, 1993.

[4] American Society for Testing and Materials (ASTM), Standard Terminology Relating to Vehicle-Pavement Systems, ASTM Standard E867-06, West Conshohocken, Pennsylvania, 2012.

[5] Federal Highway Administration (FHWA), Highway Performance Monitoring System (HPMS) Field Manual, US Department of Transportation, Washington, DC, 2015.

[6] Federal Highway Administration (FHWA), Highway Performance Monitoring System (HPMS) Field Manual, US Department of Transportation, Washington, DC, 2016.

[7] Federal Highway Administration (FHWA), National Performance Measurement Measures: Highway Safety Improvement Program, Title 23 Code of Federal Regulations (CFR) Part 490, US Department of Transportation, Washington, DC, 2016.

[8] M. W. Sayers, T. D. Gillespie, and C. A. V. Querioz, The International Road Roughness Experiment: Establishing Correlation and a Calibration Standard for Measurements, World Bank Technical Paper No. 45, Washington, DC, 1986 .

[9] D. J. Sheskin, Handbook of Parametric and Nonparametric Statistical Procedures, Chapman and Hall/CRC, Boca Raton, Florida, 2011. 